\title{
Childhood cognitive function and adult psychopathology: associations with psychotic and non-psychotic symptoms in the general population
}

Jennifer H. Barnett, Fiona McDougall, Man K. Xu, Tim J. Croudace, Marcus Richards and Peter B. Jones

\section{Background}

Lower cognitive ability in childhood is associated with increased risk of future schizophrenia, but its relationship with adult psychotic-like experiences and other psychopathology is less understood.

\section{Aims \\ To investigate whether this childhood risk factor is shared with adult subclinical psychiatric phenotypes including psychotic-like experiences and general psychiatric morbidity.}

\section{Method}

A population-based sample of participants born in Great Britain during 1 week in March 1946 was contacted up to 20 times between ages 6 weeks and 53 years. Cognition was assessed at ages 8, 11 and 15 years using a composite of age-appropriate verbal and non-verbal cognitive tests. At age 53 years, psychotic-like experiences were self-reported by 2918 participants using four items from the Psychosis Screening Questionnaire and general psychiatric morbidity was assessed using the scaled version of the General Health Questionnaire (GHQ-28).

\section{Results}

Psychotic-like experiences were reported by $22 \%$ of participants, and were highly comorbid with other psychopathology. Their presence in adults was significantly associated with poorer childhood cognitive test scores at ages 8 and 15 years, and marginally so at age 11 years. In contrast, high GHQ scores were not associated with poorer childhood cognition after adjustment for the presence of psychotic-like experiences.

\section{Conclusions}

Psychotic and non-psychotic psychopathologic symptoms are highly comorbid in the general population. Lower childhood cognitive ability is a risk factor for psychotic-like experiences in mid-life; these phenomena may be one end of a continuum of phenotypic expression driven by variation in early neurodevelopment.

\section{Declaration of interest}

J.H.B. is an employee of Cambridge Cognition Ltd and co-inventor on patent PCT/GB2005/003279 (methods for assessing psychotic disorders) with P.B.J.
Relative to their peers, individuals who will later develop schizophrenia show subtle cognitive decrements throughout childhood and adolescence. ${ }^{1,2}$ This decrement is estimated to average six IQ points, equating to a $4 \%$ increase in risk of schizophrenia for every one point lower IQ. ${ }^{3}$ Lower IQ score is one of the most replicated risk factors for schizophrenia; it is remarkably consistent across populations and is a key piece of evidence arguing that schizophrenia is a neurodevelopmental disorder. ${ }^{4,5}$ The association between premorbid cognitive decrements and other psychiatric disorders is less clear. ${ }^{6-8}$ For example, in the 1946 British birth cohort, also known as the National Survey of Health and Development (NSHD), lower childhood cognitive scores have been associated with increased risk of a wide range of adult psychopathology, including schizophrenia, affective illness and alcohol misuse. ${ }^{2,9,10}$ However, there is considerable comorbidity between psychotic and other psychiatric symptoms in the general population. ${ }^{11-13}$ As a result it is not clear whether lower childhood IQ score is associated specifically with risk of schizophrenia or more broadly with risk of psychotic symptoms of any duration and intensity - or, indeed, whether it indicates a general predisposition to mental distress.

Individuals who meet criteria for a clinical diagnosis of schizophrenia represent one extreme of a spectrum of psychotic experiences; larger proportions of the population experience similar phenomena at a lower intensity or frequency. Since the majority of individuals are not distressed or impaired by them, we follow the example of the Avon Longitudinal Study of Parents and Children (ALSPAC) in referring to the milder phenomena as 'psychotic-like experiences. ${ }^{14}$ The prevalence of such experiences reported by a population varies considerably, depending on sampling methods, the form and wording of the questions used and demographic factors such as age. ${ }^{15}$ Using self-report instruments, around a quarter of adults typically endorse screening questions for psychotic-like experiences, ${ }^{16-19}$ whereas only a small minority (1-4\%) concurrently meet diagnostic criteria for a psychotic disorder.

It is not known whether the underlying aetiology of psychoticlike experiences is the same as that of clinical psychosis. Studying the developmental history and antecedents of such experiences requires prospective population-based data in order to avoid potential selection bias, and to ensure that risk factors are assessed prior to - and independently of - psychiatric outcome. This study investigated whether childhood cognitive function is associated with subclinical adult psychotic-like experiences in the NSHD and, if so, whether it is specific to psychotic-like experiences or also (independently) associated with more general subclinical psychopathology. We predicted that poorer childhood cognitive function would be seen among adults who reported psychotic-like experiences, but not among adults who reported depression and anxiety but no psychotic-like experience.

\section{Method}

The NSHD is a prospective cohort study of individuals born in England, Scotland and Wales in one week in March 1946. The 
cohort was stratified for social class such that all births to the wives of agricultural and non-manual workers in that week were included, along with one in four births to the wives of manual workers, the overwhelming occupational group in post-war Britain. The sample excluded multiple births and births outside marriage. Data have been collected from the sample on 20 occasions, most recently in 1999, when its members were 53 years old. ${ }^{20}$ Ethical approval for this study was granted by the North Thames multicentre research ethics committee, and all participants gave informed consent.

\section{Adult symptom assessment}

At the age 53 assessment participants rated their psychotic-like experiences over the previous 12 months using five screening-level items from the Psychosis Screening Questionnaire (PSQ). ${ }^{21}$ These questions have been used in other British adult population studies to establish the prevalence of broadly defined psychotic-like experiences. ${ }^{19}$ As in previous surveys, the hypomania item ('Have there been times when you felt very happy indeed without a break for days on end?') was endorsed by a large proportion (51.5\%) of respondents $(n=1506)$, with a further $643(22.0 \%)$ rating themselves as 'unsure'; as a result of this apparent lack of specificity, this item was dropped. Responses to the four remaining PSQ items, referring to symptoms of thought interference, persecution, strange experiences and hallucinations were coded as positive if the individual definitely endorsed that item, and negative if the symptom was absent or the respondent was unsure. General psychiatric morbidity was also self-rated at the age 53 assessment using the 28-item version of the General Health Questionnaire (GHQ). ${ }^{22}$ Patients rated their experience over the previous few weeks of symptoms such as 'lost much sleep over worry'. A four-point scale was used, and a total score was calculated (range 29-109, mean 45.3, s.d.=9.6). High scorers were defined as individuals in the 85th percentile or above (GHQ score of 53 or more); this cut-off was chosen to represent individuals scoring approximately 1 standard deviation above the mean in a theoretical normal distribution.

Clinically relevant psychotic symptoms were investigated at age 36 years using a shortened version of the Present State Examination (PSE). ${ }^{20,23}$ This nurse-led interview included ten items probing for delusions and hallucinations over the previous month. Items were considered positive if symptoms were present and judged clinically relevant during that period. This represents a more narrowly defined and rarer measure of psychotic experience at an earlier age; we used these data in a sensitivity analysis to investigate the possible effect of information bias on the primary results regarding psychotic-like experiences at age 53 years.

\section{Childhood cognitive assessment}

Age-appropriate verbal and non-verbal cognitive tests were conducted at ages 8 years, 11 years and 15 years. $^{24,25}$ Principal components analyses were conducted separately at each age on the available cognitive measures. These comprised: at age 8 years, picture intelligence, reading, sentence completion and vocabulary; at age 11 years, arithmetic, reading, vocabulary, and verbal and non-verbal IQ from the AH4 Group Test of Intelligence; ${ }^{26}$ and at age 15 years, mathematics, reading, and AH4 verbal and nonverbal IQ. First principal component scores explained respectively $74 \%, 76 \%$ and $74 \%$ of variance in cognitive test scores at ages 8 , 11 and 15 years; these scores were used as the summary cognitive measure for all subsequent analyses.

\section{Early-life socioeconomic factors}

Two early-life socioeconomic factors were considered as possible confounders for an association between childhood IQ score and adult psychopathologic symptoms: occupational group of father at birth (classified according to ten categories) and material home circumstances at age 4 years, a nine-level variable aggregated from health visitor ratings of the condition of clothing and shoes, and the condition and occupancy level of the participant's dwelling.

\section{Statistical analysis}

Chi-squared tests were used to compare PSQ symptom frequencies between men and women, to assess associations between the presence of PSQ symptoms and the two socioeconomic variables, and to assess associations of PSQ symptoms endorsement (any/none) with high GHQ score ( $\geqslant 53)$. Linear regression with log-transformed GHQ score as the dependent variable and the number of PSQ symptoms endorsed (range $0-4)$ as the predictor was used to determine whether there was a dose-response relationship between adult psychotic-like experiences and general psychiatric morbidity.

Mean cognitive scores at each childhood assessment were compared using independent $t$-tests between groups defined by the presence or absence of one or more positive responses in separate analyses for PSQ and PSE and using normal/high GHQ score $(<53$ or $\geqslant 53)$. To quantify the converse relationship, cognitive scores (separately at each age) were used to predict the presence or absence of PSQ symptoms in a logistic regression. To differentiate the relationships between childhood cognition and psychotic-like experiences from those between childhood cognition and general non-psychotic psychiatric morbidity, linear regressions were conducted at each of the three ages with cognitive score as the dependent variable and two binary variables (presence/absence of PSQ symptoms and GHQ score $\geqslant 53$ ) entered simultaneously as independent predictors. Our final model included effects of PSQ score (presence/absence), GHQ score (high/normal), paternal occupation, material home circumstances, gender, and gender $\times$ PSQ and gender $\times$ GHQ interaction terms. Linear regressions with cognitive scores as the dependent variables and the number of PSQ symptoms endorsed (range 0-4) as the predictor were used to determine whether there was a dose-response relationship between childhood cognition and adult psychotic-like experiences. Finally, we inspected the distributions of childhood cognitive test scores (as density plots) in order to ascertain whether any effects were due to a subgroup of individuals with high or low scores, or whether they might be due to shifts in the whole distribution of scores between groups with and without symptoms.

\section{Results}

The PSQ was completed by 2918 participants at age 53 years. Major reasons for non-response included death $(n=469)$, temporary or permanent refusal $(n=920)$, participants who were temporarily or permanently abroad $(n=580)$ or who could not be traced $(n=330)$. Excluding those who had died or were abroad, $68 \%$ responded. The four psychotic-like experiences were positively endorsed with the following prevalences: thought interference $n=302$ (10.3\%); persecution $n=397$ (13.6\%); strange experiences $n=216$ (7.4\%); hallucinations $n=107$ (3.7\%). Less than $1 \%$ of participants endorsed all four symptoms. Symptom frequencies were equal in men and women $\left(\chi^{2}=2.92\right.$, d.f. $=3$, $P=0.4)$ and endorsement of one or more symptoms was unrelated 
to paternal occupation $\left(\chi^{2}=9.09\right.$, d.f. $\left.=9, P=0.4\right)$ or material home circumstances $\left(\chi^{2}=5.71\right.$, d.f. $\left.=8, P=0.7\right)$.

\section{Childhood cognition and adult psychotic-like experiences}

Childhood cognitive scores were significantly lower among the $651(22.3 \%)$ individuals who endorsed any psychotic-like experience at age 53 years than among the 2265 individuals who did not: age 8 years $t=3.93$, d.f. $=2586, P<0.0001$, mean difference $z=0.18$ (95\% CI $0.09-0.27)$; age 11 years $t=3.09$, d.f. $=2478, P=0.002$, mean difference $z=0.14$ (95\% CI 0.05 $0.23)$; age 15 years $t=3.43$, d.f. $=2475, P=0.0006$, mean difference $z=0.16$ (95\% CI 0.07-0.25); Fig. 1(a). When the relationship was modelled in the opposite direction using logistic regression, the presence of psychotic-like experiences at age 53 years was significantly predicted by cognitive scores at ages 8 years (odds ratio $(\mathrm{OR})=0.83,95 \%$ CI $0.75-0.91, z=-3.9, P=0.0001), 11$ years $(\mathrm{OR}=0.86,95 \% \mathrm{CI} 0.78-0.95, z=-3.08, P=0.002)$ and 15 years $(\mathrm{OR}=0.84,95 \%$ CI $0.76-0.93, z=-3.42, P=0.0006)$. Among the $164(5.0 \%)$ individuals who experienced clinically rated psychotic symptoms at age 36 years according to the PSE, the same pattern emerged: age 8 years $t=2.75$, d.f. $=2908, P=0.006$, mean difference $z=0.23$ (95\% CI 0.07-0.39); age 11 years $t=2.41$, d.f. $=2805, P=0.02$, mean difference $z=0.20$ (95\% CI 0.04 0.37 ); age 15 years $t=3.42$, d.f. $=2796, P=0.0006$, mean difference $z=0.29$ (95\% CI 0.12-0.45); Fig. 1(b).

\section{Childhood cognition and other psychopathology}

High levels of general psychiatric morbidity (defined as a GHQ score $\geqslant 53)$ were reported by $489(16.9 \%)$ of survey members. There was a highly significant overlap between individuals in the high GHQ group and those endorsing PSQ symptoms: $n=398(13.8 \%)$ of the sample; $\chi^{2}=246.0$, d.f. $=1, P \ll 0.0001$. In linear regression the number of PSQ symptoms endorsed was also a highly significant predictor of GHQ score: $r^{2}=0.13, \beta=0.36$, $t=20.8, P \ll 0.0001$ (Fig. 2). Compared with the majority of the sample, cognitive scores were lower in the high GHQ group at ages 8 years $(t=2.4$, d.f. $=2564, P=0.02)$ and 15 years $(t=3.3$, d.f. $=2462, P=0.001)$ but not at age 11 years $(t=1.9$, d.f. $=2464$, $P=0.06)$; Fig. 1(c).

\section{Separating psychotic and non-psychotic effects}

When entered simultaneously into a linear regression, presence or absence of PSQ symptoms was a significant predictor of childhood cognitive scores at all ages (age 8 years $\beta=-0.07, t=-3.3$, $P=0.001$; age 11 years $\beta=-0.05, t=-2.6, P=0.01$; age 15 years $\beta=-0.06, t=-2.6, P=0.009$ ), whereas GHQ score (high $v$. normal) was only a significant predictor of cognitive score at age 15 years $(\beta=-0.05, t=-2.3, P=0.02)$. Mean cognitive scores for groups defined by both psychotic-like experience status (present/absent) and GHQ status (high/normal) are shown in Fig. 3. At all three ages scores showed a consistent pattern across groups, with highest scores in those with no symptoms and lowest in those who reported both psychotic-like experiences and high GHQ scores. Analysis by $t$-test confirmed that, among individuals with high GHQ scores, cognitive scores were significantly lower in the 226 who reported psychotic-like experiences at age 53 years than in the 217 who did not at age 8 years $(t=2.21$, d.f. $=441$, $P=0.027$, mean difference $z=0.20,95 \%$ CI $0.02-0.38)$ and at 15 years ( $t=2.40$, d.f. $=406, P=0.017$, mean difference $z=0.22$, 95\% CI 0.04-0.40), although not at age 11 years $(t=1.44$, d.f. $=420, P=0.15$, mean difference $z=0.14,95 \%$ CI $0.05-0.33)$.

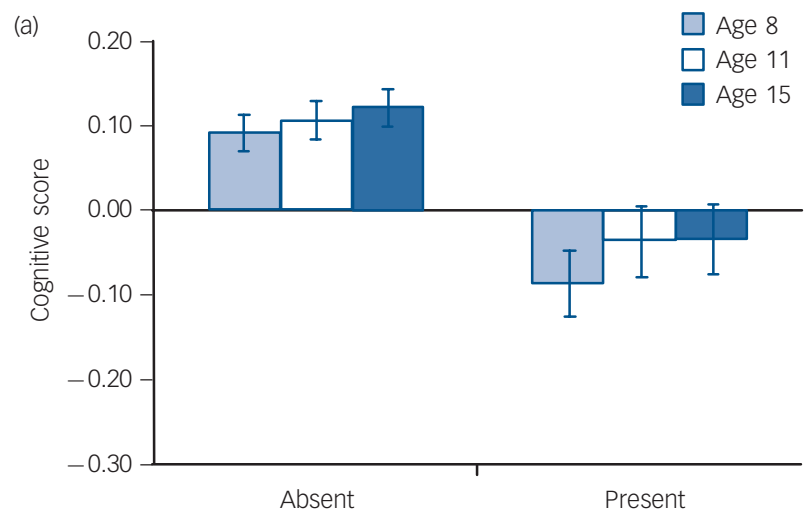

PSQ psychotic symptoms at age 53

(b)

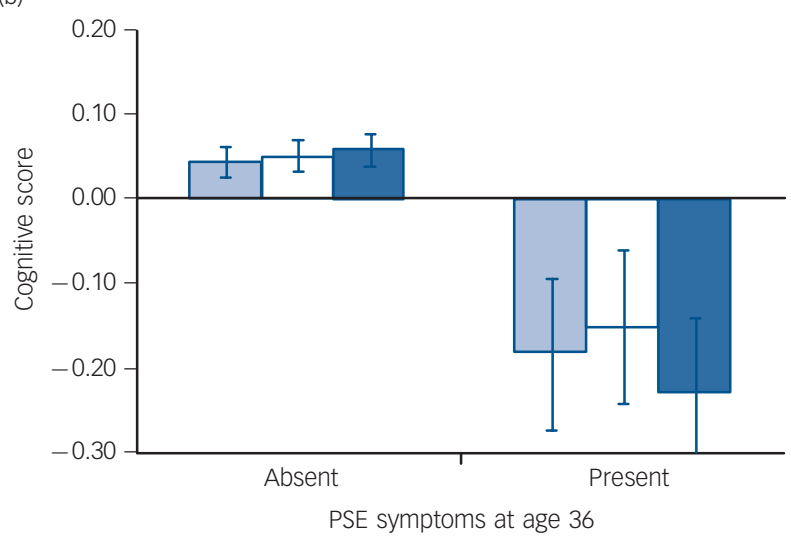

(c)

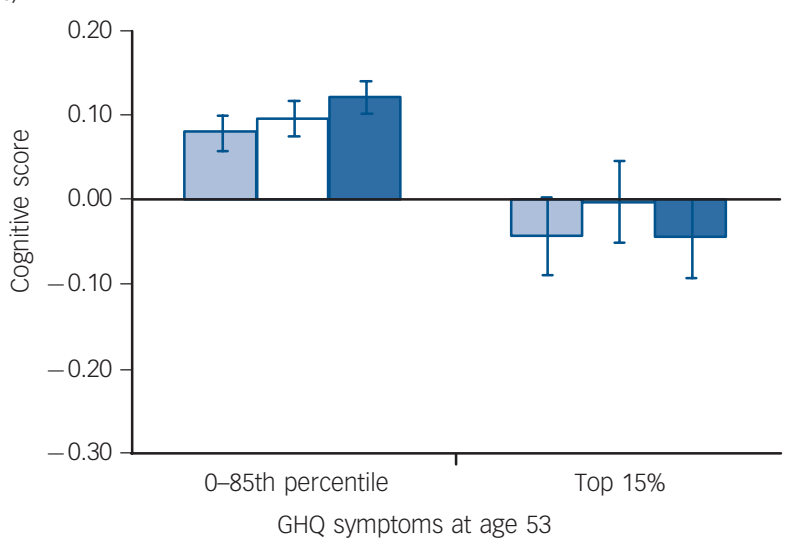

Fig. 1 Childhood cognitive scores among members of the 1946 British birth cohort, analysed by (a) psychotic-like experiences at age 53 years (Psychosis Screening Questionnaire, PSQ); (b) clinically relevant psychotic symptoms at age 36 years (Present State Examination, PSE); and (c) normal v. high general psychiatric morbidity at age 53 years (General Health Questionnaire, GHQ). Scores are mean values, bars indicate standard error.

Our most complete model included terms for PSQ and GHQ scores, socioeconomic measures, gender and gender $\times$ symptom interaction terms. The PSQ score had a significant effect on cognitive score at each age (all $P<0.05$ ), whereas GHQ score had no significant effect at any age $(P>0.05)$. Gender was a significant predictor of cognitive score at age 15 years only $(F=8.48$, d.f. $=1,2445, P=0.003)$ but not at ages 8 or 11 years $(P>0.05)$. There was no significant interaction between gender and PSQ or GHQ symptoms (all $P>0.05$ ). Both paternal occupation and material home circumstances had significant effects on cognitive score at all three ages (all $P \ll 0.0001)$. 


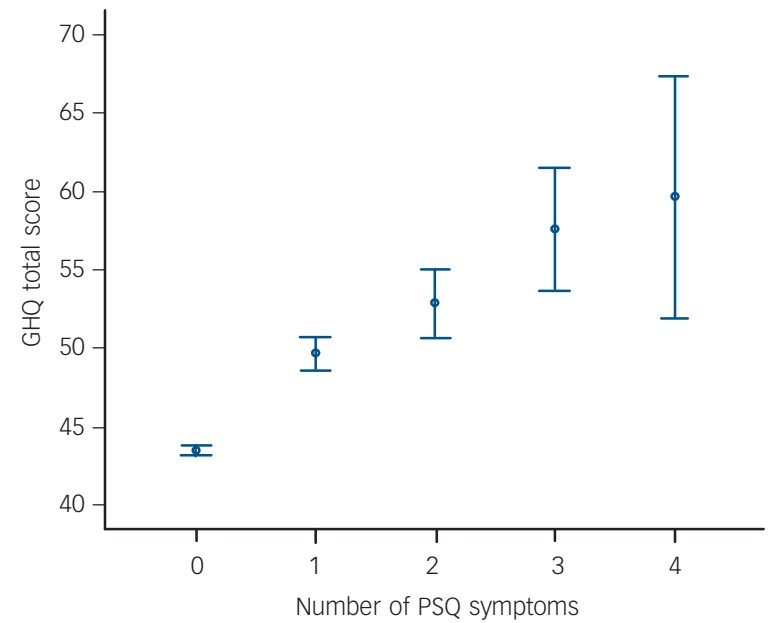

Fig. 2 General Health Questionnaire (GHQ) score for each level of Psychosis Screening Questionnaire (PSQ) symptom endorsement at age 53 years $(n=2280)$. Scores are mean values, bars indicate $95 \%$ confidence intervals.

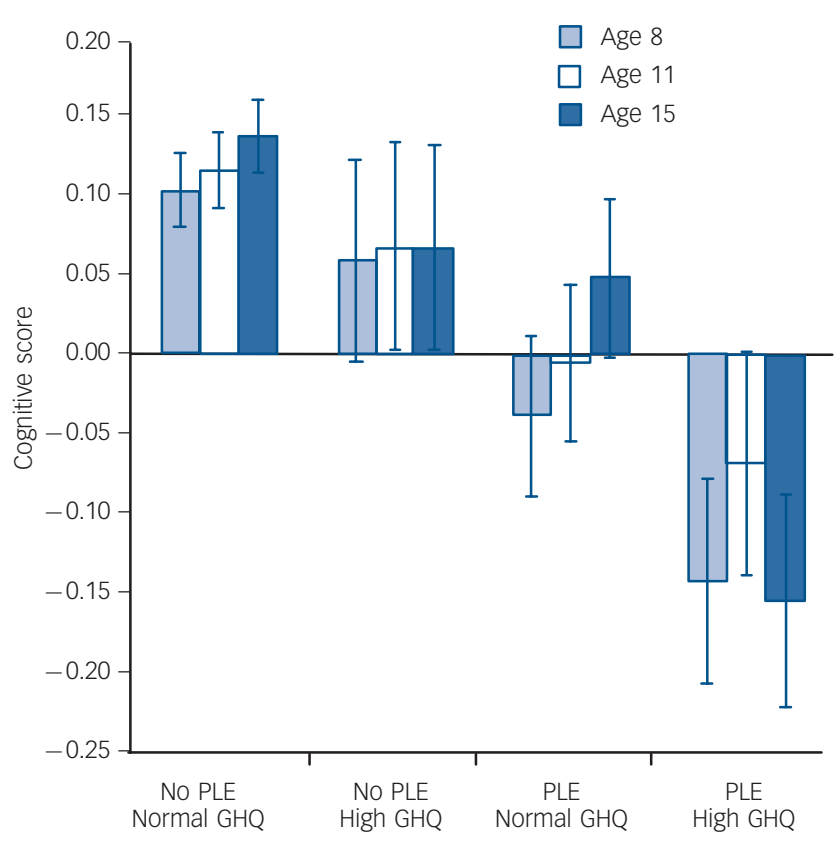

Fig. 3 Childhood cognitive scores according to presence $v$. absence of self-reported psychotic-like experience (PLE) and high v. normal General Health Questionnaire (GHQ) psychopathology score at age 53 years. Scores are mean values, bars indicate standard error

\section{Number and type of psychotic-like experiences}

Linear regression confirmed a dose-response relationship between number of psychotic-like experiences endorsed and cognitive scores at ages 8 years $(\beta=-0.08, t=-3.8, P=0.0001), 11$ years $(\beta=-0.06, \quad t=-2.9, \quad P=0.003)$ and 15 years $(\beta=-0.07$, $t=-3.5, P=0.0004)$. Mean cognitive scores were lowest among those who endorsed all four PSQ symptoms (Fig. 4); however, post hoc $t$-tests across all levels of symptoms showed that the only significant group differences were lower cognitive scores in those endorsing one symptom compared with those endorsing no symptoms $(P<0.05$ at ages 8,11 and 15 years). To determine

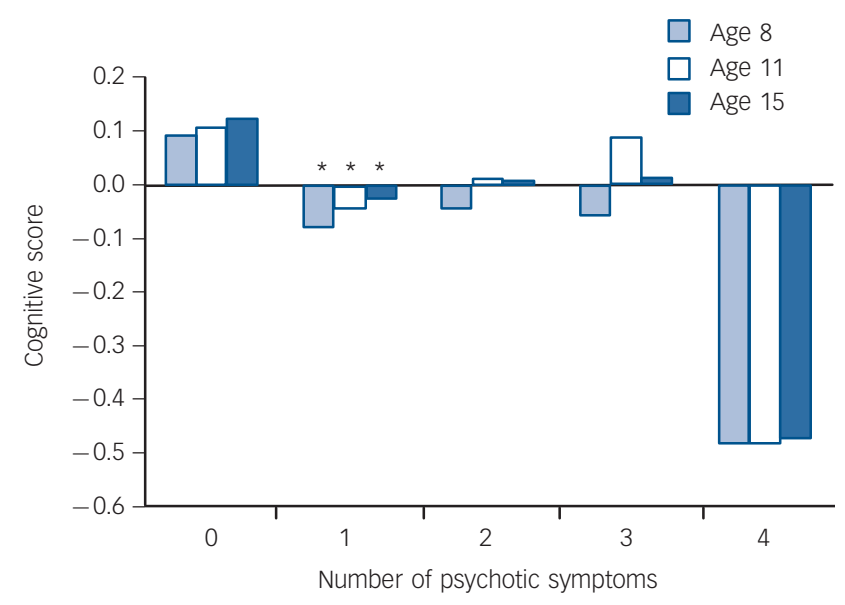

Fig. 4 Childhood cognitive scores according to number of

Psychosis Screening Questionnaire symptoms endorsed at age 53 years. Asterisks indicate significant post hoc differences in mean cognitive score $(P<0.05)$ compared with individuals endorsing no symptoms which of the four symptoms was most predictive of cognitive score, linear regression was conducted with cognitive score at each age as the dependent variable and each of the four symptoms (present/absent) entered simultaneously as predictors. 'Hallucinations' was the only item consistently associated with cognitive score (at age 8 years $\beta=-0.05, t=-2.5, P=0.01$; age 11 years $\beta=-0.07, t=-3.1, P=0.002$; age 15 years $\beta=-0.06$, $t=-3.0, P=0.003$; all models fit at $P<0.01)$. Of the other symptoms, only 'strange experiences' was additionally associated with cognition, and that only at age 15 years $(\beta=-0.05$, $t=-2.0, P=0.04)$. Mean cognitive scores categorised by presence or absence of each PSQ symptom type are shown in Fig. 5.

\section{Distribution of cognitive scores}

One important consideration is whether the lower group means of those who later reported psychotic-like experiences are due to a minority of individuals with very low cognitive scores. Visual inspection of the distribution of cognitive scores suggests that this is not the case, i.e. that the difference between populations who do and do not experience psychotic-like experiences is largely due to a small leftward shift in the entire population (Fig. 6). Interestingly, the same is broadly true of the distributions of childhood cognitive scores among the small minority (5\%) of individuals who experienced clinically relevant symptoms at age 36 years (Fig. 6(b)).

\section{Discussion}

Using data from a unique population-based cohort, we show that scores from general ability tests in childhood and adolescence predict the likelihood of experiencing both subclinical psychotic-like experiences and clinically relevant psychotic symptoms in middle age, as they do schizophrenia. General psychiatric morbidity in mid-life was highly comorbid with psychotic-like experiences, and we believe it is this comorbidity that drives previously reported associations between childhood cognition and non-psychotic psychopathology. In this sample, although GHQ scores were also associated with poorer childhood cognitive development, ${ }^{10}$ the association appeared to be driven primarily by psychotic-like experiences: once such experiences 

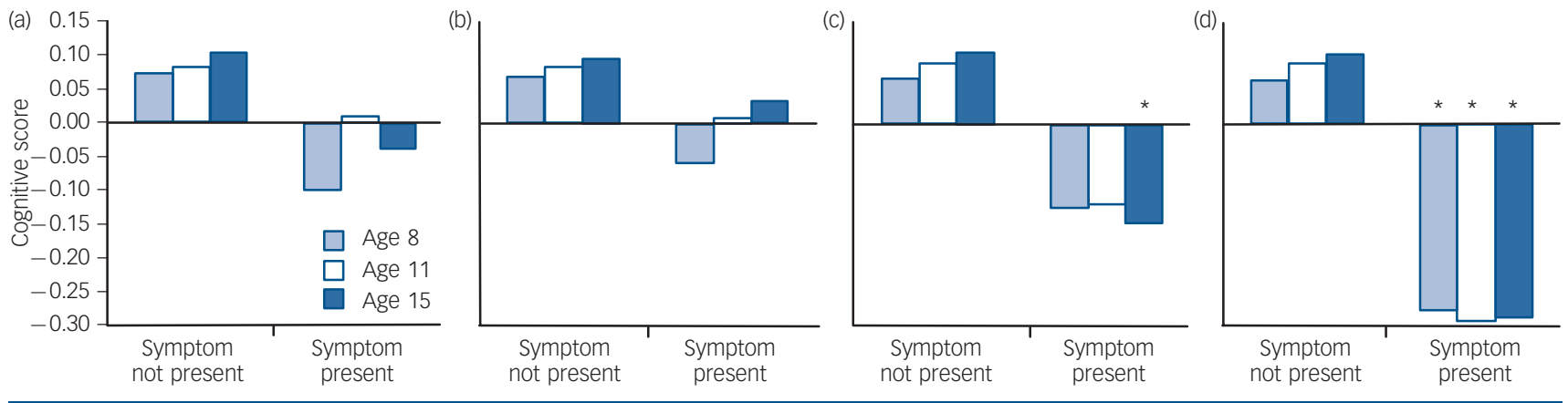

Fig. 5 Childhood cognitive scores (mean values) according to individual psychotic-like experiences reported at age 53 years: (a) thought interference; (b) persecution; (c) strange experiences; (d) hallucinations. Asterisks indicate symptoms providing a significant independent contribution to the prediction of childhood cognitive score $(P<0.05)$.

(a)
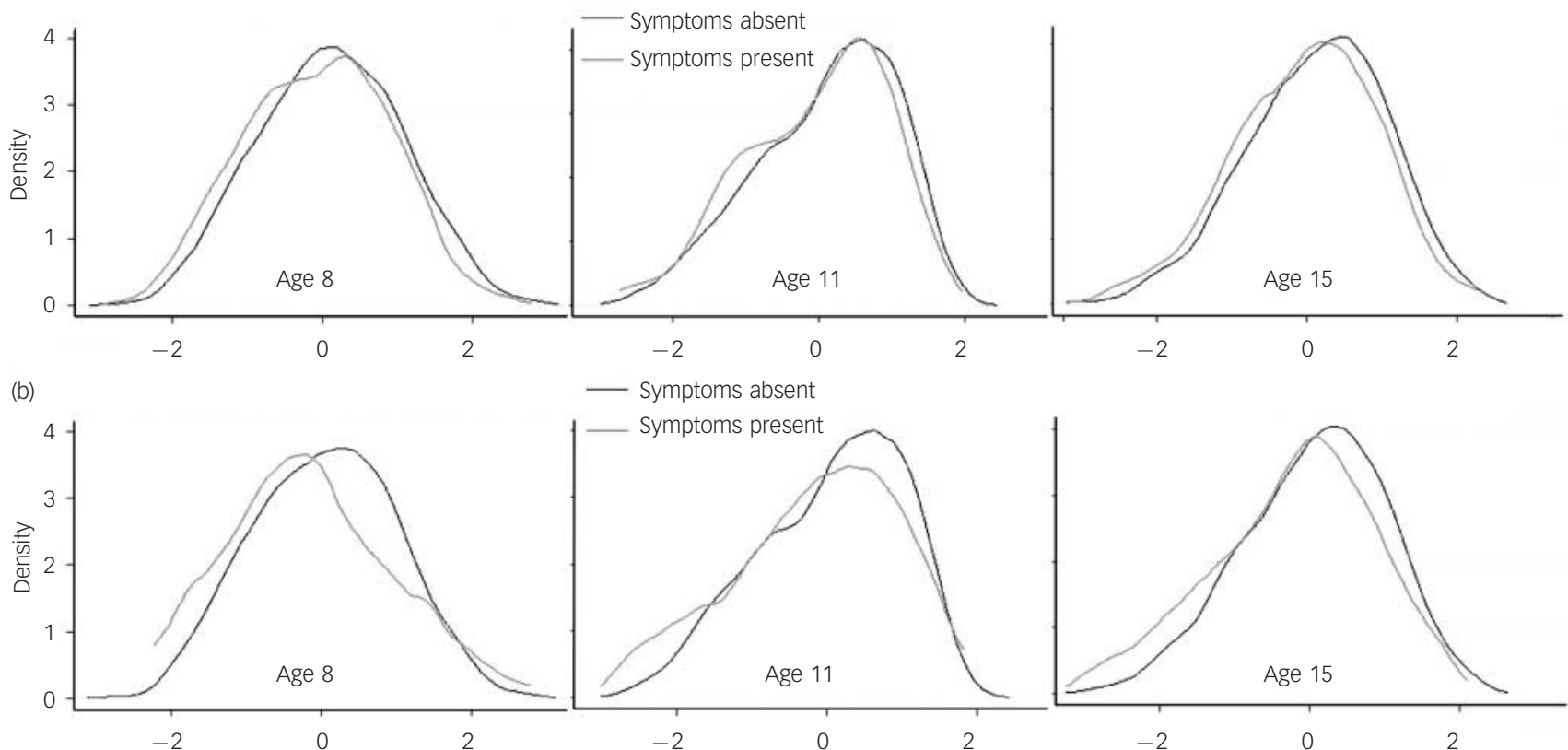

Fig. 6 Density plot of population distribution of childhood cognitive scores: (a) presence/absence of psychotic-like experiences (Psychosis Screening Questionnaire) at age 53 years; (b) clinically relevant psychotic symptoms (Present State Examination) at age 36 years.

were controlled for, the association between GHQ score and childhood cognition was strikingly removed. This does not suggest that psychotic-like experiences and GHQ scores are not correlated, merely that they may be mechanistically different in terms of their relationship with cognition.

We believe these results suggest that the pathophysiology of subclinical psychotic-like experiences may be consistent with that of clinically relevant psychosis and neurodevelopmental in origin, and that this is somewhat in contrast to other forms of psychiatric morbidity. Our current understanding of the pathophysiology of schizophrenia posits that developmental differences reflect aberrance in early brain development, the full effects of which do not become apparent until late adolescence or adulthood., ${ }^{4,28}$ The presence of early cognitive decrements among individuals who will later experience psychotic-like experiences may suggest that this model applies more broadly throughout the spectrum of risk for psychosis. It is also possible that some of the cognitive decrement could reflect an early static injury (e.g. caused by fetal hypoxia) rather than aberrance in postnatal developmental trajectory. Indeed, such effects may be caused by a number of interacting factors including genetic predisposition, prenatal and perinatal factors such as maternal infection and obstetric complications, ${ }^{29,30}$ and later environmental effects such as substance use and social isolation. ${ }^{31,32}$ This model, which has been used primarily to explain rare, clinically relevant psychotic disorder, may therefore be applicable to a wider range of the population and to the principles of the wide range of normal childhood development. For example, studies of childhood psychotic-like experiences in the Bristol-based ALSPAC birth cohort have shown them to be associated with schizophrenia risk factors including lower childhood IQ score, low birth weight and prenatal events such as maternal infection and diabetes. ${ }^{14,33,34}$

\section{Strengths and limitations}

The unique nature of this sample allows several other methodological explanations for the associations between adult 
psychotic-like experiences and childhood cognition to be excluded. Our main analyses stemmed from self-report responses from a small number of broad questions about psychotic-like experiences, and it is possible that individuals with lower cognitive scores might be more likely to misinterpret or misread questions about such experiences in self-report questionnaires. However, this explanation seems unlikely given that the associations between childhood cognition and self-reported PSQ scores are mirrored by those found 16 years previously during an interview administered by a trained nurse (and indeed, by those found in patients with a medical history consistent with an adult diagnosis of schizophrenia). ${ }^{2}$ This previous case ascertainment also allows us to estimate the extent to which cognitive scores in the group reporting psychotic-like experiences were influenced by individuals who, 10 years earlier, had at any time during adulthood met criteria for a diagnosis of schizophrenia: 16 such individuals completed the PSQ at age 53 years, only 3 of whom reported psychotic-like experiences at that age. The availability of sociodemographic variables from early life is an additional strength of this sample, confirming that this assumed neurodevelopmental effect was unlikely to be driven by parental social class or home circumstances during early childhood.

The large sample size allows characterisation of the cognitive score distribution in the minority of individuals who experienced psychotic-like experiences as well as the majority who did not; inspection of these distributions (Fig. 6) suggests that the effects are not driven by a small number of outliers but reflect a population-wide shift in the efficiency of neurodevelopment. This model is supported by our finding of a dose-response relationship between cognitive scores and number of PSQ symptoms endorsed (see Fig. 4), suggesting that the mean cognitive scores would decrease among individuals located along the continuum of psychotic experiences, from no psychotic-like experience at one end, to early-onset schizophrenia at the other. Adult psychotic-like experiences may in this respect differ from those found in childhood and adolescence, since in the ALSPAC study, risk of psychotic-like experiences at age 12 years was reported to be associated with IQ score in a non-linear manner, with no decrease in risk seen in children with above-average IQ scores. ${ }^{14}$ Similarly, and in contrast to the associations reported in schizophrenia, ${ }^{35}$ the relationship between childhood cognition and adult psychotic-like experiences did not differ between men and women.

A further limitation of the study is that individuals may have had psychotic-like experiences at times outside of the PSQ assessment window. Thus, the no-symptom group may contain some false negatives, which would lead to a conservative bias in the effects we describe. Conversely, since these experiences are more common during adolescence and early adulthood than in later years, ${ }^{36}$ individuals who report them in late adulthood may represent a 'poor prognosis' subset whose experiences did not dissipate with age, exacerbating the association between childhood cognition and late-life experiences. Similarly, sample attrition would be expected to lead to underrepresentation both of individuals with poorer cognitive function and of individuals with psychotic symptoms, potentially producing a conservative bias. Wadsworth et al conducted an in-depth analysis of factors that predicted non-response at age 53 years: these included male gender, low cognitive test score at age 8 years, behaviour at age 15 years and educational, stress and social factors during early adulthood. ${ }^{20}$ It is likely that some of these factors may also be related to the likelihood of experiencing psychotic-like episodes. Thus, despite the generally improved representativeness of birth cohort studies when compared with other forms of sample selection, the prevalence of psychotic-like experiences reported here may have been influenced by attrition.

\section{Implications}

A notable feature of this study is the high degree of comorbidity found between adult psychotic-like experiences and GHQ scores. Although our analyses suggest that psychotic-like experiences are the primary feature driving previously reported associations between childhood cognitive scores and adult anxiety and depression, ${ }^{9,10}$ it is nonetheless interesting that among those with the highest levels of general psychiatric morbidity, approximately half simultaneously reported psychotic-like experiences. It is plausible that mid-life psychotic-like experiences may be an expression of the same underlying anxiety and depression trait (or traits) measured by the GHQ, the expression of which as a psychotic-like experience might be determined in part by cognitive factors.

In conclusion, cognitive scores in childhood, previously shown to predict adult schizophrenia, were here shown to predict a range of subsyndromal psychotic experiences in the general population at age 53 years. As in schizophrenia, the distribution of cognitive scores suggests that the association between childhood IQ level and risk of psychosis reflects not a subgroup of severely impaired individuals, but rather a subtle shift in the efficiency of neurodevelopment among those who will later experience psychotic-like experiences. Although this shift is too subtle to be of diagnostic value, it suggests that the neurodevelopmental aberrance that underlies schizophrenia is also present from early in life among individuals in the broader psychosis spectrum.

Jennifer H. Barnett, PhD, Department of Psychiatry, University of Cambridge Cambridgeshire \& Peterborough Foundation National Health Service (NHS) Trust and Cambridge Cognition Ltd, Cambridge; Fiona McDougall, PhD, Man K. Xu, DPhil, Tim J. Croudace, PhD, Department of Psychiatry, University of Cambridge and Cambridgeshire \& Peterborough Foundation NHS Trust: Marcus Richards, PhD, Medical Research Council (MRC) National Survey of Health and Development, MRO Unit for Lifelong Health and Ageing, Psychiatry, University of Cambridge, Cambridgeshire \& Peterborough Foundation NHS Trust, National Institute for Health Research Collaboration for Leadership in Applied Health Research and Care for Cambridgeshire \& Peterborough, and MRC/Wellcome Trust Behavioural and Clinical Neurosciences Institute, Cambridge, UK

Correspondence: Dr J. Barnett, Department of Psychiatry, Addenbrooke's Hospital, Cambridge CB2 2QQ, UK. Email: jhb32@cam.ac.uk

First received 30 Aug 2011, final revision 16 Mar 2012, accepted 3 Apr 2012

\section{Funding}

The study was supported by Wellcome Trust grant 088869/Z/09/Z. M.R. was supported by the Medical Research Council. TJ C. was supported by a National Institute for Health Research (NIHR) Career Scientist Award in Public Mental Health. This work forms part of the NIHR Collaboration for Leadership in Applied Health Research \& Care for Cambridgeshire \& Peterborough and the NIHR Cambridge Biomedical Research Centre.

\section{References}

1 Aylward E, Walker E, Bettes B. Intelligence in schizophrenia: meta-analysis of the research. Schizophr Bull 1984; 10: 430-59.

2 Jones $\mathrm{P}$, Rodgers $\mathrm{B}$, Murray $\mathrm{R}$, Marmot $\mathrm{M}$. Child development risk factors for adult schizophrenia in the British 1946 birth cohort. Lancet 1994; 344: 1398-402.

3 Khandaker GM, Barnett JH, White IR, Jones PB. A quantitative meta-analysis of population-based studies of premorbid intelligence and schizophrenia. Schizophr Res 2011; 132: 220-7.

4 Murray RM, Lewis SW. Is schizophrenia a neurodevelopmental disorder? BMJ 1987; 295: 681-2

5 Murray RM, Sham P, van Os J, Zanelli J, Cannon M, McDonald C. A developmental model for similarities and dissimilarities between schizophrenia and bipolar disorder. Schizophr Res 2004; 71: 405-16.

6 Cannon M, Caspi A, Moffitt TE, Harrington H, Taylor A, Murray RM, et al. Evidence for early-childhood, pan-developmental impairment specific to schizophreniform disorder: results from a longitudinal birth cohort. Arch Gen Psychiatry 2002; 59: 449-56. 
7 Koenen KC, Moffitt TE, Roberts AL, Martin LT, Kubzansky L, Harrington $\mathrm{H}_{\text {, }}$ et al. Childhood IQ and adult mental disorders: a test of the cognitive reserve hypothesis. Am J Psychiatry 2009; 166: 50-7.

8 Reichenberg A, Weiser M, Rabinowitz J, Caspi A, Schmeidler J, Mark M, et al. A population-based cohort study of premorbid intellectual, language, and behavioral functioning in patients with schizophrenia, schizoaffective disorder, and nonpsychotic bipolar disorder. Am J Psychiatry 2002; 159: 2027-35.

9 Van Os J, Jones P, Lewis G, Wadsworth M, Murray R. Developmental precursors of affective illness in a general population birth cohort. Arch Gen Psychiatry 1997; 54: 625-31.

10 Hatch SL, Jones PB, Kuh D, Hardy R, Wadsworth ME, Richards M. Childhood cognitive ability and adult mental health in the British 1946 birth cohort. SoC Sci Med 2007; 64: 2285-96.

11 Freeman $D$, McManus S, Brugha T, Meltzer H, Jenkins R, Bebbington $P$. Concomitants of paranoia in the general population. Psychol Med 2011; 41 923-36.

12 Wigman JTW, Lin A, Vollebergh WAM, van Os J, Raaijmakers QA, Nelson B, et al. Subclinical psychosis and depression: co-occurring phenomena that do not predict each other over time. Schizophr Res 2011; 130: 277-81.

13 Varghese D, Scott J, Welham J, Bor W, Najman J, O'Callaghan M, et al. Psychotic-like experiences in major depression and anxiety disorders: a population-based survey in young adults. Schizophr Bull 2011; 37: 389-93.

14 Horwood J, Salvi G, Thomas K, Duffy L, Gunnell D, Hollis C, et al. IQ and non-clinical psychotic symptoms in 12-year-olds: results from the ALSPAC birth cohort. Br J Psychiatry 2008; 193: 185-91.

15 Linscott RJ, van Os J. Systematic reviews of categorical versus continuum models in psychosis: evidence for discontinuous subpopulations underlying a psychometric continuum. Implications for DSM-V, DSM-VI, and DSM-VII. Annu Rev Clin Psychol 2010; 6: 391-419.

16 Kendler KS, Gallagher TJ, Abelson JM, Kessler RC. Lifetime prevalence, demographic risk factors, and diagnostic validity of nonaffective psychosis as assessed in a US community sample. The National Comorbidity Survey. Arch Gen Psychiatry 1996; 53: 1022-31.

17 Poulton R, Caspi A, Moffitt TE, Cannon M, Murray R, Harrington H. Children's self-reported psychotic symptoms and adult schizophreniform disorder: a 15-year longitudinal study. Arch Gen Psychiatry 2000; 57: 1053-8.

18 Van Os J, Hanssen M, Bijl RV, Ravelli A. Strauss (1969) revisited: a psychosis continuum in the general population? Schizophr Res 2000; 45: 11-20.

19 Johns LC, Cannon M, Singleton N, Murray RM, Farrell M, Brugha T, et al. Prevalence and correlates of self-reported psychotic symptoms in the British population. Br J Psychiatry 2004; 185: 298-305

20 Wadsworth M, Kuh D, Richards M, Hardy R. Cohort profile: the 1946 National Birth Cohort (MRC National Survey of Health and Development) Int J Epidemiol 2006; 35: 49-54.
21 Bebbington $\mathrm{P}$, Nayani T. The psychosis screening questionnaire. Int $J$ Methods Psychiatr Res 1995; 5: 11-9.

22 Goldberg DP, Hillier VF. A scaled version of the General Health Questionnaire. Psychol Med 1979; 9: 139-45.

23 Wing J, Cooper J, Sartorius N. The Description and Classification of Psychiatric Symptoms; An Instruction Manual for the PSE and CATEGO System. Cambridge University Press, 1974.

24 Pigeon DA. Tests used in the 1954 and 1957 surveys. In The Home and The School (ed JWB Douglas): 129-32. Macgibbon \& Kee, 1964.

25 Pigeon DA. Details of the fifteen years tests. In All Our Future (eds JWB Douglas, JM Ross, HR Simpson): 194-7. Davies, 1968.

26 Heim AW. The AH4 Group Test of Intelligence. nferNelson, 1970.

27 Weinberger DR. Implications of normal brain development for the pathogenesis of schizophrenia. Arch Gen Psychiatry 1987; 44: 660-9.

28 Rapoport JL, Addington AM, Frangou S. The neurodevelopmental model of schizophrenia: update 2005. Mol Psychiatry 2005; 10: 434-49.

29 Boksa P. Effects of prenatal infection on brain development and behavior: a review of findings from animal models. Brain Behav Immunity 2010; 24: $881-97$.

30 Cannon M, Jones PB, Murray RM. Obstetric complications and schizophrenia: historical and meta-analytic review. Am J Psychiatry 2002; 159: 1080-92.

31 Moore THM, Zammit S, Lingford-Hughes A, Barnes TR, Jones PB, Burke M, et al. Cannabis use and risk of psychotic or affective mental health outcomes: a systematic review. Lancet 2007; 370: 319-28.

32 Coid JW, Kirkbride JB, Barker D, Cowden F, Stamps R, Yang M, et al. Raised incidence rates of all psychoses among migrant groups: findings from the East London first episode psychosis study. Arch Gen Psychiatry 2008; 65: 1250-8

33 Thomas K, Harrison G, Zammit S, Lewis G, Horwood J, Heron J, et al. Association of measures of fetal and childhood growth with non-clinical psychotic symptoms in 12-year-olds: the ALSPAC cohort. Br J Psychiatry 2009; 194: 521-6.

34 Zammit S, Odd D, Horwood J, Thompson A, Thomas K, Menezes P, et al. Investigating whether adverse prenatal and perinatal events are associated with non-clinical psychotic symptoms at age 12 years in the ALSPAC birth cohort. Psychol Med 2009; 39: 1457-67.

35 Weiser M, Reichenberg A, Rabinowitz J, Kaplan Z, Mark M, Nahon D, et al. Gender differences in premorbid cognitive performance in a national cohort of schizophrenic patients. Schizophr Res 2000; 45: 185-90.

36 Scott J, Chant D, Andrews G, McGrath J. Psychotic-like experiences in the general community: the correlates of CIDI psychosis screen items in an Australian sample. Psychol Med 2006; 36: 231-8. 